Revue

d'ethnoécologie
Revue d'ethnoécologie

6 | 2014

Conservation de la nature : quel rôle pour les sciences sociales?

\title{
Conservation de la nature : quel rôle pour les sciences sociales?
}

Vers une anthropologie de la conservation

Conservation of nature: what role for social sciences? Towards an anthropology

of conservation

Richard Dumez, Marie Roué et Serge Bahuchet

\section{(2) OpenEdition}

Journals

Édition électronique

URL : http://journals.openedition.org/ethnoecologie/2089

DOI : $10.4000 /$ ethnoecologie.2089

ISSN : 2267-2419

Éditeur

Laboratoire Eco-anthropologie et Ethnobiologie

Référence électronique

Richard Dumez, Marie Roué et Serge Bahuchet, « Conservation de la nature : quel rôle pour les sciences sociales? », Revue d'ethnoécologie [En ligne], 6 | 2014, mis en ligne le 31 décembre 2014, consulté le 19 avril 2019. URL : http://journals.openedition.org/ethnoecologie/2089; DOI : 10.4000/ ethnoecologie.2089

Ce document a été généré automatiquement le 19 avril 2019

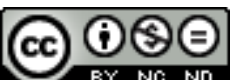

Revue d'ethnoécologie est mis à disposition selon les termes de la licence Creative Commons Attribution - Pas d'Utilisation Commerciale - Pas de Modification 4.0 International. 


\title{
Conservation de la nature : quel rôle pour les sciences sociales?
}

\author{
Vers une anthropologie de la conservation \\ Conservation of nature: what role for social sciences? Towards an anthropology \\ of conservation
}

Richard Dumez, Marie Roué et Serge Bahuchet

1 En décembre 2007, grâce au soutien du MNHN et de l'INEE $^{1}$ du CNRS, nous avons réuni au Musée de l'Homme des chercheurs internationaux en sciences sociales, mais aussi en sciences naturelles et des acteurs impliqués dans l'étude des processus de conservation et de gestion de la biodiversité, pour réfléchir ensemble, à partir d'expériences réelles, à la question: «Conservation de la nature : quel rôle pour les sciences sociales?». Notre atelier s'était donné comme objectif d'analyser, non pas les conditions de la coexistence, mais les questions scientifiques posées aux chercheurs par cette coexistence entre les sociétés et la biodiversité. Une partie des textes de ce numéro sont issus de ce colloque international.

2 Ce questionnement a été engagé beaucoup plus tôt dans le domaine de l'écologie. La biologie de la conservation $\mathrm{a}$, dès la fin du $20^{\mathrm{e}}$ siècle tenté de répondre à une situation de crise environnementale. La conservation de la biodiversité devient alors un des objectifs majeurs des sciences de la nature. Michael E. Soule a fait émerger le champ scientifique de la Conservation Biology au début des années 1980 (Soule \& Wilcox 1980, Soule 1985) que Robert Barbault a popularisé ensuite en France. Dans le Dictionnaire de l'écologie, en 1999, à l'item « Protection des espèces », R. Barbault définit la biologie de la conservation, cette « discipline de crise » de l'extinction actuelle des espèces animales et végétales, comme :

« une nouvelle discipline de synthèse qui applique les principes de l'écologie, de la biogéographie, de la génétique des populations, de l'anthropologie, de l'économie, de la sociologie, etc., au maintien de la diversité biologique sur l'ensemble de la planète » (Barbault 2001 : 1127) ${ }^{2}$

3 précisant que les dénominations «sciences de la conservation» ou "écologie de la conservation » seraient peut-être préférables. La biologie de la conservation est pourtant 
toujours aujourd'hui une discipline des sciences de la vie, et donc menée principalement par des biologistes et des écologues.

Cependant les débats sont vifs quant aux choix et aux stratégies de conservation de la biodiversité. Ils portent tant sur leur efficacité que sur des questions d'équité et de justice sociale. Pour qui conserver (Bahuchet et al. 2000) ? Quels sont les usages « légitimes » de la nature et ceux qui ne le sont pas? Quels sont les liens entre biodiversité et diversité culturelle (Roué 2006) ? Comment analyser la durabilité de certaines pratiques qui transforment le milieu tout en le gérant (Dumez 2010) ? En bref, la question de la relation complexe entre biodiversité et société, et même entre biodiversité et diversité culturelle, est au centre de nos interrogations. Nous proposons dans ce numéro de la Revue d'ethnoécologie d'en débattre afin de poser les bases d'une anthropologie de la conservation qui vienne prêter main forte à la biologie de la conservation.

5 Ce n'est en effet un secret pour personne, en tous cas pour aucun anthropologue, que les décisions de conservation génèrent fort fréquemment des conflits avec les habitants. L'association entre conservation et développement durable, qui un temps a semblé indispensable, a donné lieu à la fondation de programmes intégrés (IPCD ${ }^{3}$ ), qui sont maintenant généralement contestés, au point que le volet «développement » en est fréquemment abandonné (Newmark \& Hough 2000). C'est peu de dire que les populations humaines sont « un problème » pour les spécialistes de la biodiversité, et que les moyens pour le surmonter ne font pas consensus (cf. Bahuchet \& McKey 2005). Même si une partie des spécialistes de la biologie de la conservation commencent à prendre conscience du fait que «les actions de conservation sont des comportements humains» («Conservation actions are ultimately human behaviors » Fox et al. 2006: 1817), un long chemin reste à parcourir.

6 Parmi les solutions pour conserver un espace et des espèces, la plus ancienne et la plus classique est la délimitation d'aires intégralement protégées, exclusives de toute implantation humaine, dont l'archétype est le Parc national de Yellowstone, aux USA. Basé sur le mythe de la "nature vierge", de la wilderness, un concept construit culturellement aux États-Unis dans un contexte religieux (Nash 1982, Cronon 1995, Roué 2006, Diegues 2008), il est toujours bien vivant malgré sa dénonciation récurrente. À l'autre extrême, la conservation dans les zones habitées de ce que l'on nomme « la nature ordinaire ", appelle des processus différents. La conservation porte alors sur les espèces ou sur les écosystèmes, sans que l'homologie entre l'un et l'autre soit nécessairement clairement discutée.

7 Les sciences sociales, et en particulier l'anthropologie, s'engagent fortement dans les problématiques de la conservation et des problèmes qu'elle pose (Ghimire \& Pimbert 1997, Monde \& Razon 1999). Un courant académique fort inventif s'est développé autour de la notion de political ecology (Robbins 2004). Les recherches sur les savoirs locaux et autochtones suscitent également un grand nombre de recherches fondamentales ou appliquées, souvent en relation avec des initiatives de co-gestion ou de gestion participative. Enfin l'ecological history démontre à quel point les écosystèmes les plus célébrés pour leur diversité "sauvage» (tels l'Amazonie, Balée 1994, 1998) ont été fortement investis pendant des millénaires par des sociétés qui, loin de les exploiter sans vergogne, ont transformé ces milieux, voire accru leur biodiversité. Tous ces mouvements et données peuvent-ils ou non permettre l'émergence d'une "anthropologie de la conservation »? 
8 L'ethnoécologie et l'anthropologie de la nature s'intéressent à la place de l'homme dans son environnement naturel : comment les sociétés interagissent-elles avec ce dernier? Quels savoirs et pratiques naturalistes possèdent-elles? Comment vivent-elles et se représentent-elles la nature (cf. Posey 1999) ? Ainsi, se dessinent non pas une nature mais des natures, et de multiples ontologies, ou modes de relations entre humains et nonhumains (vivants ou non) qui dépassent la dichotomie nature-culture (cf. Descola 2005). Si la « crise d'extinction » de multiples espèces touche directement les sciences biologiques (Barnosky et al. 2011), elle interroge aussi les sciences sociales.

9 La relation entre la société et la biodiversité, cet objet scientifique aux dimensions à la fois théoriques et concrètes (au sens de la diversité génétique, spécifique et écosystémique), soulève en effet de multiples interrogations :

- sur la place de l'Homme dans son environnement: comment et dans quelles mesures contribue-t-il à "créer de la biodiversité ", à tout le moins des mosaïques paysagères qui accroissent la biodiversité ? Dans quelle mesure les pratiques des sociétés conservent-elles ou menacent-elles la biodiversité?

- sur les actions à mener : conserver, cogérer, piloter (cf. Blandin 2009)?

- sur les interactions sociales entre les populations locales et les acteurs de la conservation et sur les questions de gouvernance et d'éthique qui leurs sont associées;

- sur les espaces concernés, entre espaces protégés et nature ordinaire, entre paysages naturels et paysages anthropiques;

- sur les pratiques et les savoirs naturalistes des populations locales et leur transmission, sur leur impact sur la biodiversité et sa gestion.

L'ethnoécologie et l'anthropologie de la nature se sont donné pour tâche d'explorer ces questions. Pourtant nous préconisons ici la nécessité d'une véritable « anthropologie de la conservation ». Un tel courant, ancré dans le champ des sciences sociales, nait de la confluence de deux approches scientifiques, celle de la biologie de la conservation, et celle de l'ethnoécologie et de l'anthropologie de la nature. Mais l'anthropologie de la conservation ne se résume pas au seul objectif de conserver de la biodiversité. L'anthropologie de la conservation cherche, avec les outils et méthodes propres à l'anthropologie et à l'ethnoécologie, à comprendre la relation complexe entre biodiversité et diversité culturelle, en complémentarité avec la biologie de la conservation. S'il y a confluence entre biologie de la conservation et anthropologie de la conservation, il n'y a pourtant en effet pas totale convergence entre les objectifs de ces deux champs de recherche. Là où les forces de la biologie de la conservation sont projetées vers une nécessaire réflexion et vers une action pour une conservation de la biodiversité, l'anthropologie de la conservation va s'attacher à deux dimensions de la conservation de la nature.

11 La première dimension, qui converge avec la biologie de la conservation, répond à une demande sociétale: les éléments constitutifs de la diversité biologique, en tant que patrimoine de l'Humanité, doivent être préservés autant pour leur valeur intrinsèque que pour leur valeur extrinsèque. Ces éléments de nature sont en effet également pour les sociétés des éléments de culture. Ils représentent non seulement des ressources naturelles, mais également un mode de vie, en relation avec une culture et une éthique particulières à chaque société. L'article $8 \mathrm{j}$ de la Convention sur la diversité Biologique (1992) met l'accent sur le respect des communautés autochtones et locales qui apparaissent, par leurs savoirs et leurs pratiques, comme des acteurs potentiels de la conservation et de l'utilisation durable de la biodiversité. Les savoirs locaux et 
autochtones (souvent dénommés en anglais TEK, traditional ecological knowledge ou ILK, indigenous and local knowledge) sont un champ de recherche en fort développement depuis plusieurs décennies. Ces savoirs, qui permettent aux sociétés locales de penser et d'agir sur le monde naturel, sont à la base de leur système de gestion. Toute proposition de conservation se doit donc de tenir compte de ces savoirs, tant pour des raisons éthiques que par souci d'efficacité.

La seconde dimension de l'anthropologie de la conservation peut apparaitre plus divergente en ce qu'elle fait un objet d'étude de la conservation de la nature en tant que mode de pensée et d'action, mais aussi de ceux qui sont concernés, c'est-à-dire autant ceux qui la promeuvent et mettent en œuvre (y compris les scientifiques, notamment les biologistes de la conservation) que ceux qui sont impactés par celle-ci. La mise en protection d'espèces et d'espaces a en effet une influence directe sur les populations locales, du fait de la valeur qu'elles ont pour elles, surtout lorsque ce processus de mise en protection n'est pas le fait des populations locales et qu'il n'est donc pas approprié par elles. L'anthropologie de la conservation va tout autant étudier les conséquences de la conservation pour les populations locales que les processus et interactions sociales qu'elles suscitent, ainsi que les actions et logiques discursives des acteurs de la conservation.

On le voit, l'anthropologie de la conservation doit dépasser une anthropologie des seules aires protégées, pour s'intéresser à l'ensemble de l'environnement, qu'il s'agisse de nature ordinaire ou extraordinaire, de nature en ville ou de nature "pristine", " primaire », « vierge » ou décrite comme telle.

Elle est dans le même temps une « discipline de crise », pour reprendre les mots de Robert Barbault, et une discipline qui prend ses distances par rapport au diagnostic des écologues pour s'intéresser à tous les processus d'interactions entre sociétés et nature. Elle porte tout à la fois une recherche proprement fondamentale et une recherche appliquée, au travers d'une ingénierie écologique et sociale (Roturier \& Roué 2009). Enfin, ce champ de l'anthropologie de la conservation, ouvert par l'anthropologie et l'ethnoécologie, s'ouvre et se nourrit d'autres sciences sociales : la géographie, le droit, la political ecology, etc.

Rejoignant Robert Barbault (2014), nous proposons que les sciences de la conservation deviennent un champ scientifique englobant biologie de la conservation et anthropologie de la conservation. Les chercheurs de ces deux disciplines, ou tout au moins de ces deux disciplines devrions-nous dire, qui se nourrissent l'une l'autre, collaborent alors selon un gradient allant de recherches pluridisciplinaires additionnées, où chacun étudie un même objet selon le seul prisme de sa discipline, à de véritables recherches interdisciplinaires intégrées où l'objet de recherche est le fruit d'une co-construction.

Notre colloque des 17 et 18 décembre 2007 a permis de dessiner les grands thèmes du champ que devrait couvrir l'anthropologie de la conservation, en abordant les aires protégées, les parcs et les populations locales, les espèces protégées et les espèces menacées, l'équité et la justice sociale dans les actions de conservation, la coexistence des savoirs locaux et savoirs scientifiques et la co-construction des savoirs, les expériences, les projets et les nouveaux paradigmes...

17 La présente livraison s'en fait l'écho, en publiant quelques-unes des communications, remaniées, présentées à cette occasion, mais surtout en réunissant des essais composés 
ultérieurement par de jeunes chercheurs, s'inspirant de ces discussions et les éclairant à partir de leurs propres recherches.

Nous poursuivrons ces réflexions dans les prochaines livraisons, toujours dans la continuité de notre colloque initial, mais surtout en publiant des recherches en cours et novatrices. En effet, nous voulons offrir aux chercheurs dans notre Revue d'ethnoécologie un espace privilégié pour ce nouveau champ que nous souhaitons développer, celui de l'anthropologie de la conservation.

\section{BIBLIOGRAPHIE}

Bahuchet S., Grenand F., Grenand P. \& de Maret P. 2000 - Forêts des tropiques, forêts anthropiques. Vol. I de « Les Peuples des forêts tropicales aujourd'hui ». Bruxelles, Commission européenne APFT., $132 \mathrm{p}$.

Bahuchet S. \& McKey D. 2005 - L'homme et la biodiversité tropicale. In Marty P., Vivien F.D., Lepart J. \& Larrère R. (Ed.) Les biodiversités. Objets, théories, pratiques. Paris, CNRS : 37-55.

Balée W. 1994 - Footprints of the forest: Ka'apor ethnobotany - The historical ecology of plant utilization by an Amazonian people. New York, Colombia University Press, 396 p.

Balée W. (Ed.) 1998 - Advances in historical ecology. New York, Columbia University Press, 429 p.

Barbault R. 2001 - Protection des espèces. In Dictionnaire de l'écologie. Paris, Encylopædia universalis \& Albin Michel : 1116-1129.

Barbault R. 2014 - Au nom du vivant. Plaidoyer pour réconcilier l'homme et la nature. Paris, Buchet Chastel, $112 \mathrm{p}$.

Barnosky A.D., Matzke N., Tomiya S., Wogan G.O., Swartz B., Quental T.B., Marshall C., McGuire J.L., Lindsey E.L. \& Maguire K.C. 2011 - Has the Earth's sixth mass extinction already arrived? Nature 471 (7336) : 51-57.

Blandin P. 2009 - De la protection de la nature au pilotage de la biodiversité. Versailles, Quae, 124 p. Cronon W. 1995- The Trouble with Wilderness; or, Getting Back to the Wrong Nature. In Cronon W. (Ed.) Uncommon Ground: Rethinking the Human Place in Nature. New York, Norton \& Co : 69-90.

Descola P. 2005 - Par-delà nature et culture. Paris, Gallimard, 624 p.

Diegues A.C. 2008 - 0 mito moderno da natureza intocada (6 ${ }^{\mathrm{e}}$ ed.). São Paulo, Hucitec/NUPAUB, $198 \mathrm{p}$.

Dumez R. 2010 - Le feu, savoirs et pratiques en Cévennes. Versailles, Quæ, 245 p. (Indisciplines).

Fox H.E., Christian C., Nordby J.C., Pergams O.R., Peterson G.D. \& Pyke C.R. 2006 - Perceived barriers to integrating social science and conservation. Conservation Biology, 20 (6) : 1817-1820.

Ghirmire K.B. \& Pimbert M.P. 1997 - Social Change and Conservation. UK, Earthscan Publications Limited, $342 \mathrm{p}$.

Monde J.-C. \& Razon J.-P. (Ed.) 1999 - Nature sauvage, nature sauvée ? Écologie et peuples autochtones. Paris, Peuples autochtones et développement, 235 p. (Ethnies Documents ; 24-25). 
Nash R. 1982 - Wilderness and the American Mind, 3rd ed. New Haven, Connecticut, Yale Univ. Press, $426 \mathrm{p}$.

Newmark W.D. \& Hough J.L 2000 - Conserving wildlife in Africa: integrated conservation and development projects and beyond. BioScience 50 (7) : 585-592.

Posey D.A. (Ed.) 1999 - Cultural and Spiritual Values of Biodiversity. London: United Nations Environmental Programme \& Intermediate Technology Publications, 731 p. www.unep.org/pdf/ Cultural_Spiritual_thebible.pdf

Robbins P. 2004 - Political ecology: a critical introduction. Blackwell Malden, MA, Pub., xxi + 242 p. Roturier S. \& Roué M. 2009 - Of forest, snow and lichen : Sami reindeer herders' knowledge of winter pastures in northern Sweden. Forest Ecology and Management 258 (9) : 1960-1967.

Roué M. 2006 (Ed.), Diversité culturelle et biodiversité, Revue Internationale des sciences sociales RISS, numéro 187

Roué M. 2006 - Le désert, le wilderness et la forêt : Éthique protestante et naissance de l'écologisme. In Beck C., Luginbühl Y., Muxart T. Temps et espaces des crises de l'environnement. Versailles, Quæ, 412 p. (Indisciplines).

Soule M.E. 1985 - What Is Conservation Biology? BioScience, 35 (11) : 727-734.

Soule M.E. \& Wilcox B.A. (Ed.) 1980 - Conservation biology: an evolutionary ecological perspective. Sunderland, Massachusetts, Sinauer Associates, $x v+395$ p.

\section{NOTES}

1. Institut National Écologie et Environnement.

2. La notion est déjà définie dans la première édition de 1999.

3. Integrated Projects of Conservation and Development.

\section{AUTEURS}

\section{RICHARD DUMEZ}

Maître de conférences du Muséum en ethnoécologie Muséum national d'histoire naturelle (Musée de l'Homme) UMR 7206 Éco-anthropologie et ethnobiologie Sorbonne Universités

\section{MARIE ROUÉ}

Directrice de recherches émérite, CNRS/MNHN

Laboratoire éco-anthropologie et ethnobiologie, Musée de l'Homme 17 place du Trocadéro 75016 Paris 


\section{SERGE BAHUCHET}

Professeur du Muséum

MNHN, Musée de l'Homme, Laboratoire éco-anthropologie et ethnobiologie

17 place du Trocadéro 75016 Paris 